\title{
Development of a Gas Filled Magnet spectrometer coupled with the Lohengrin spectrometer for fission study
}

\author{
G. Kessedjian ${ }^{1}$, A. Chebboubi ${ }^{1}$, H. Faust ${ }^{2}$, U. Köster ${ }^{2}$, T. Materna ${ }^{3}$, C. Sage ${ }^{1}$, O. Serot ${ }^{4}$ \\ ${ }^{1}$ LPSC, CNRS/IN2P3, UJF Grenoble 1, INPG, Grenoble, France \\ ${ }^{2}$ Institut Laue-langevin, Grenoble, France \\ ${ }^{3}$ DSM/IRFU/SPhN, CEA Saclay, France \\ ${ }^{4}$ DEN/DER/SPRC/LEPh , CEA Cadarache, France
}

\begin{abstract}
The accurate knowledge of the fission of actinides is necessary for studies of innovative nuclear reactor concepts. The fission yields have a direct influence on the evaluation of the fuel inventory or the reactor residual power after shutdown. A collaboration between the ILL, LPSC and CEA has developed a measurement program on fission fragment distributions at ILL in order to measure the isotopic and isomeric yields. The method is illustrated using the ${ }^{233} \mathrm{U}(\mathrm{n}, \mathrm{f})^{98} \mathrm{Y}$ reaction.

However, the extracted beam from the Lohengrin spectrometer is not isobaric ions which limits the low yield measurements. Presently, the coupling of the Lohengrin spectrometer with a Gas Filled Magnet (GFM) is studied at the ILL in order to define and validate the enhanced purification of the extracted beam. This work will present the results of the spectrometer characterisation, along with a comparison with a dedicated Monte Carlo simulation especially developed for this purpose.
\end{abstract}

\section{Fission yield study for the fuel cycles}

For innovative fuel cycles, nuclear charge and mass distributions of the fission products are strongly needed for three main reasons: the spent fuel inventory, the spent fuel residual heat and the prompt gamma rays emitted per actinide. For the last point, a theoretical approach is being developed with several calculation codes (GEF [1], Fifrelin [2] ...) to predict the prompt gamma ray emission for all actinides of interest. Nevertheless, these calculations are largely dependent on the fission yields, the excitation energy distributions and the spin distributions of the fission fragments. Nowadays this last important parameter is probably the least known.

Unfortunately, the fission modelling doesn't allow a precise assessment of the fission yields. This new measurement program with the Lohengrin facility aims to complete the description of the dynamical effect of fission reaction by giving the yield per isotope, excitation energy and spin:

$$
Y\left(A, Z, E^{*}, J\right)=Y(A, Z) \cdot P\left(E^{*}, J \mid A, Z\right)
$$

where $P\left(E^{*}, J \mid A, Z\right)$ is the spin distribution at excitation energy $E^{*}$. This information is needed to compare and improve the dynamical effect deduced from the microscopic approach. Experimentally, with the lohengrin spectrometer, we can determine the fission yields per mass, isotope and kinetic 
energy $Y\left(A, Z, E_{k}\right)$. The third part presents a method to provide the characteristics of the spin distribution for some isotope.

Today, the Lohengrin spectrometer is already the most powerful mass separator of fission fragments $(\triangle \mathrm{A} / \mathrm{A}=400)$ with the most important thermal neutron flux in the world $\left(5 \times 10^{15} \mathrm{n} /\left(\mathrm{cm}^{2}\right.\right.$ s)). Nevertheless, the extracted beam is not pure isobaric fission fragments since the magnetic and electric fields permit few combinations of mass, kinetic energy and ionic charge. Our collaboration is working on the coupling of the Lohengrin spectrometer with a Gas Filled Magnet (GFM) spectrometer to purify the extracted beam in order to obtain an isobaric beam. This development is presented in the second part.

\section{Coupling of a Gas Filled Magnet with the Lohengrin spectrometer}

A fission fragment spectrometer using the Gas Filled Magnet (GFM) method was already used in the past as the "Joseph" spectrometer [3]. This concept of spectrometer uses the ionic charge $q$ dependence on the ion velocity $\vec{v}$ to select the ion as a function of the magnetic field $\vec{B}$ through the Lorentz force. The ion energy loss in the gas changes its velocity and then its ionic charge. As a consequence the trajectory is drastically dependent on macroscopic parameters: magnetic field, gas nature, pressure and radius of the magnet.

\subsection{Beam selection with the Lohengrin $\otimes$ GFM spectrometer}

The Lohengrin spectrometer first achieves a magnetic deflection according to the mass over ionic charge ratio $(\mathrm{A} / \mathrm{q})$ and an electric deflection according to the kinetic energy over ionic charge ratio $\left(E_{k} / q\right)$. Thus the extracted beam is composed of all the multiplets in agreement to these two ratios : $\left(A_{1}, E_{1}, q_{1}\right) \equiv\left(A_{2}, E_{2}, q_{2}\right) \equiv\left(A_{3}, E_{3}, q_{3}\right) \ldots$ A second magnet RED for (Reverse Energy Dispersion) can be placed afterwards to focus the beam on a tape or ionization chamber coupled to Ge clover detectors. Today this facility allows the isotopic fission yield measurement in the heavy mass region per gamma spectrometry. Nevertheless, the presence of undesired masses increases the noise in the gamma ray spectrum. Presently, the collaboration has the possibility to convert the RED magnet in a GFM spectrometer to add a new separation to the Lohengrin extracted beam.

The GFM spectrometer induces a magnetic deflection according to the magnetic rigidity. Over the whole ion path, the GFM spectrometer selects the incident masses according to the mean ratio of impulsion over ionic charge which depends on the energy loss in the gas:

$$
B<\rho>=A .<V(r) / q(r)>
$$

The dependence of the ionic charge on the ion velocity is used to spread the extracted Lohengrin masses. Moreover, the ionic charge $q$ is also function of the nuclear charge $Z$ [3]. Thus, each mass or isotope could present a singular ionic optic in the double spectrometer $\{$ Lohengrin $\otimes \mathrm{GFM}\}$. Two experiments have shown the feasibility of this new spectrometer. We present in the next part the preliminary experimental results.

\subsection{GFM Experimental observation}

A first experiment has been achieved with light fragments corresponding to $A=100, E_{k}=100 \mathrm{MeV}$ and $q=20$. Thus according to the allowed multiplets, four main masses have the same ionic optic in the Lohengrin spectrometer: $A=100,95,90$, and 85 . For other masses, the fission yields or the kinetic energy have too low probabilities to be considered. A first result shows a quite good separation and the purity is around 90 to $95 \%$ (Fig. 1.) 

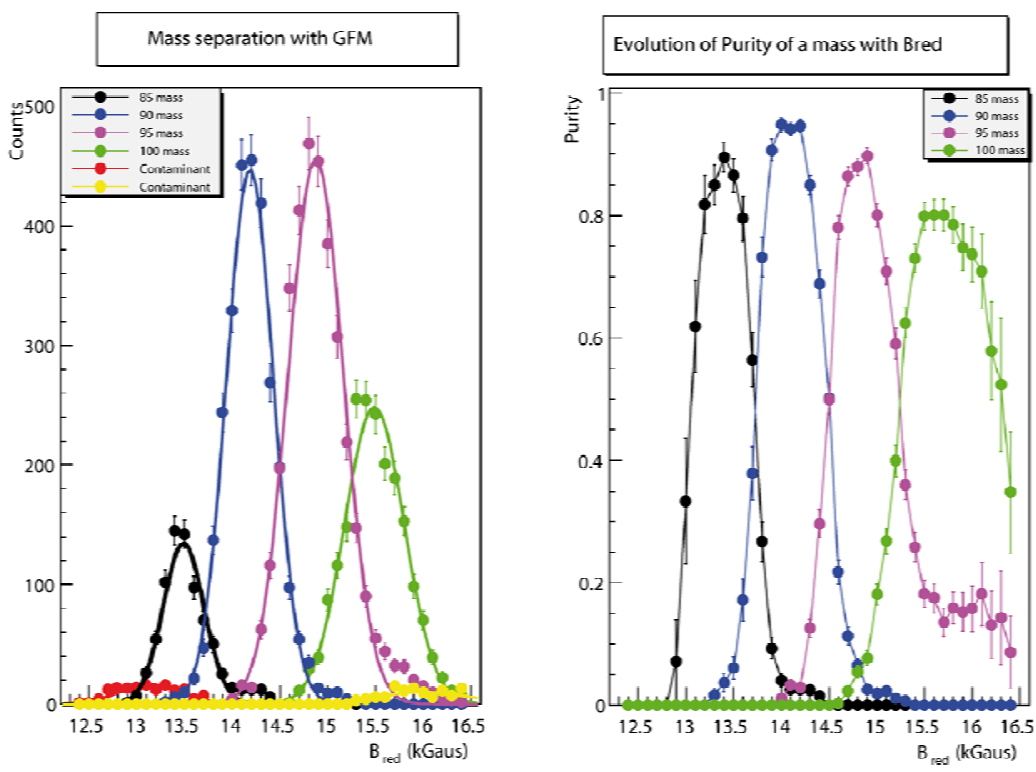

Fig. 1. Preliminary result with the $4 \mathrm{He} G F M \otimes$ Lohengrin spectrometer $\left(\mathrm{P}_{\mathrm{He}}=40 \mathrm{mbar}\right)$. (Left) Fission rate as a function of the RED magnetic field $\left(B_{\text {red }}\right)$ for a RED input beam with the parameter $A=100, E=100 \mathrm{MeV}$ and $q=20$. (Right) Purity of the extracted beam as a function of $B_{\text {red }}$.

\subsection{GFM calculations}

Monte-Carlo calculations have been realized to interpret the experimental results and characterize the acceptance of extracted beam. Initially, the GFM entrance is a $0.5 \mu \mathrm{m}$ thick polypropylene foil. Position, energy and velocity have been estimated according to the exit condition of the Lohengrin spectrometer. After the entrance foil, the effective charge is distributed $\left(\mathrm{q}_{\text {eff }}\right)$ according to the Betz model [4]. The motion equation is solved with a calculation step smaller than the mean free path of charge change $\left(l<\lambda_{q \rightarrow q}\right)$. This last parameter is determined using the electron capture or loss probabilities according to the Paul model [5]. Then the charge distribution is stochastically defined through the path in the GFM. At each step, the energy loss in the gas is deduced from the BetheBloch equation for heavy ion [6] (Fig.2).

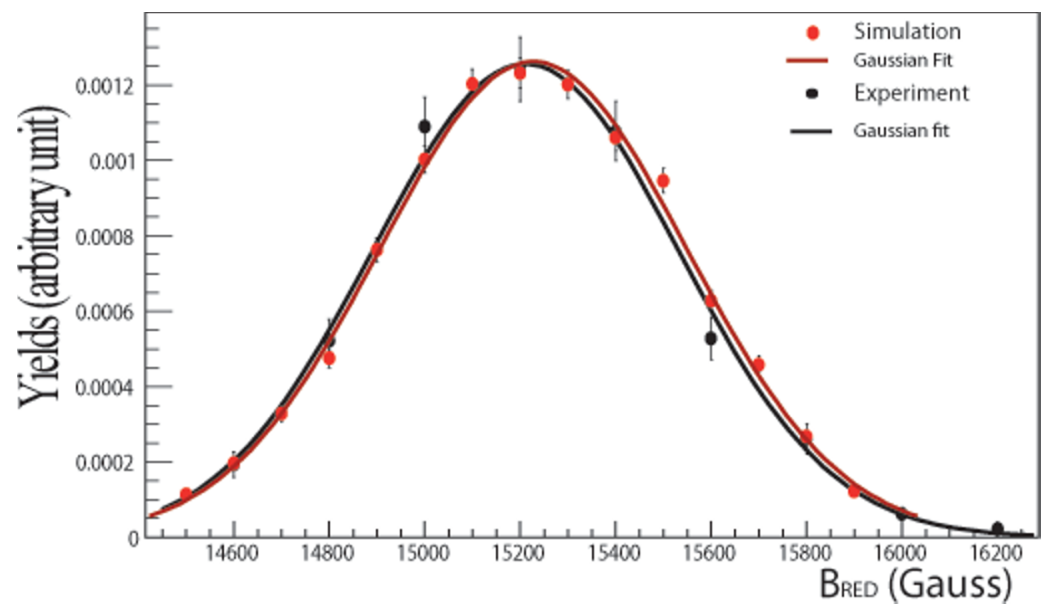

Fig. 2. Preliminary result with the $4 \mathrm{He} G F M \otimes$ Lohengrin spectrometer $\left(\mathrm{P}_{\mathrm{He}}=40 \mathrm{mbar}\right)$. In red the MonteCarlo calculation and in black the experimental data. 


\section{Isomeric fission yields on the 234U*}

The evaluation of fragment spin can be done via the measurement of isomeric yields $Y\left(A, Z, E^{*}, J\right)$, which fully characterize a fission event. Based on the statistical model for instance, the spin population function $\mathrm{P}(\mathrm{J})$ as function of mass $\mathrm{A}$, charge $\mathrm{Z}$ and excitation energy $\mathrm{E}^{*}$ can be deduced from experiment [7]. The isomeric fission yields at mean kinetic energy can be measured with the Lohengrin spectrometer of the ILL with the use of a gamma ray array of germanium detectors and an ionization chamber. Such a measurement was achieved for some isotopes of the ${ }^{233} \mathrm{U}\left(\mathrm{n}_{\mathrm{th}}, \mathrm{f}\right)$ reaction: ${ }^{88} \mathrm{Br} ;{ }^{94} \mathrm{Y},{ }^{98} \mathrm{Y} ;{ }^{99} \mathrm{Y} ;{ }^{129} \mathrm{Sb} ;{ }^{132} \mathrm{Te}$. Table 1 shows preliminary results of the isomeric yield ratios for ${ }^{98} \mathrm{Y}$ at the mean kinetic energy $\sim 92 \mathrm{MeV}$.

Table 1. Ratios of Isomeric fission yields for the ${ }^{233} \mathrm{U}(\mathrm{n}, \mathrm{f}){ }^{98} \mathrm{Y}$ reaction at the $92 \mathrm{MeV}$ mean kinetic energy with the absolute uncertainties.

\begin{tabular}{|c|c|c|c|c|c|}
\hline$\underset{J^{\pi}}{\text { Isomeric Ratio (t=tof) }}$ & $\begin{array}{c}\text { GS } \\
0^{-}\end{array}$ & $\begin{array}{l}2.0 \mathrm{~s} \\
(4,5)\end{array}$ & $\begin{array}{c}0.62 \mu \mathrm{s} \\
2^{-}\end{array}$ & $\begin{array}{c}7.6 \mu \mathrm{s} \\
4^{-}\end{array}$ & $\begin{array}{l}0.83 \mu \mathrm{s} \\
10^{-}\end{array}$ \\
\hline GS (548s) & 1 & & & & \\
\hline m (2s) & $\begin{array}{c}0.158 \\
\pm 0.008 \\
\end{array}$ & 1 & & & \\
\hline$\mu 1(0.62 \mu \mathrm{s})$ & $\begin{array}{c}0.023 \\
\pm 0.011\end{array}$ & $\begin{array}{c}0.146 \\
\pm 0.071\end{array}$ & 1 & & \\
\hline$\mu 2(7.6 \mu s)$ & $\begin{array}{c}0.409 \\
\pm 0.018 \\
\end{array}$ & $\begin{array}{c}2.585 \\
\pm 0.078 \\
\end{array}$ & $\begin{array}{l}17.8 \\
\pm 8.6\end{array}$ & 1 & \\
\hline$\mu 3(0.83 \mu s)$ & $\begin{array}{c}0.014 \\
\pm 0.001\end{array}$ & $\begin{array}{c}0.087 \\
\pm 0.008\end{array}$ & $\begin{array}{c}0.60 \\
\pm 0.29\end{array}$ & $\begin{array}{c}0.034 \\
\pm 0.001\end{array}$ & 1 \\
\hline
\end{tabular}

\section{Conclusions}

A description of the dynamical effect of fission reaction is provided with the fission yield per isotope, excitation energy and spin. The Ge clovers coupled to the Lohengrin mass separator allow investigating the isotopic and isomeric fission yields of fissile nuclei. Nevertheless this method is limited by the gamma ray background due to the presence of undesired masses in the beam. Thus an upgrade of the spectrometer consists of the coupling of the GFM with the existing Lohengrin spectrometers. In the perspective where a pure isobaric beam can be achieved, new measurements could be proposed to describe the fission process and to access parameters of interest for the fuel cycle.

\section{References}

[1] K.H. Shmidt et al., NEA www.oecd-nea.org/dbdata/nds_jefdoc/jefdoc-1423.pdf.

[2] O. Litaize and O. Serot, Phys. Rev. C 82, 2010

[3] H. Lawin et al., NIM 137 (1976) 103-117

[4] H. Betz, Reviews of Modern Physics, vol.44, n 13, p. 373, July 1972

[5] M. Paul et al., NIM A, vol. 277, pp. 418-430, 1989

[6] W.R. Leo, Techniques for nuclear and particle physics experiments, Springer-Verlag, 1994

[7] A. Gilbert and A.G.W. Cameron, Canadian Journal of Physics 43 (1965) 1446 